\title{
Relationship Between the Oxygen Uptake During Cardiopulmonary Exercise Testing and Left Ventricular Function in Patients with Acute Myocardial Infarction
}

\author{
Hiroshi MARUOKA ${ }^{1}$, Kamon ImaI ${ }^{2}$, Akihito KUbOta ${ }^{1}$, Kazuhisa InOUE ${ }^{1}$, Takayuki TAGUCHI ${ }^{1}$, \\ Ken Nishihara ${ }^{1}$, Kazuhiko HarA ${ }^{1}$, Osamu FujinawA ${ }^{1}$, Mitsutoshi Uematu ${ }^{1}$, Akikazu NaKayama ${ }^{1}$, \\ Tadashi MIZOROGI $^{1}$, Koukichi EHARA ${ }^{1}$ and Kazuho HosodA ${ }^{1}$
}

${ }^{1}$ Department of Physical Therapy, School of Health and Social Services, Saitama Prefectural University, Saitama 343-8540,
Japan
${ }^{2}$ Saitama Cardiovascular and Respiratory Center, Saitama 360-0105, Japan

\begin{abstract}
The relationship between exercise capacity and left ventricular function has been evaluated in 35 patients with acute myocardial infarction (34 males and 1 female; mean age $55.5 \pm 7.1$ years). Single photon emission computed tomography (SPECT) was used to measure left ventricular function in the acute phase $(4.9 \pm 2.2$ days after onset) and the chronic phase (188.5 \pm 22.9 days after onset). More than $10 \%$ left ventricular dilatation from the acute phase to the chronic phase was defined as remodeling (RM) and the subjects were divided into 2 groups: RM and non-RM. Cardiopulmonary exercise testing was performed at 1 month (1M), 3 months (3M) and 6 months (6M) after onset. In the RM group, anaerobic threshold (AT) and peak oxygen uptake (Peak $\dot{\mathrm{V}} \mathrm{O}_{2}$ ) did not change significantly. In the non-RM group, $A T$ was $15 \pm 1(\mathrm{ml} / \mathrm{min} / \mathrm{Kg})$ at $1 \mathrm{M}, 16 \pm 2$ at $3 \mathrm{M}$ and $18 \pm 4$ at 6M. Peak $\mathrm{VO}_{2}$ was $26 \pm 3(\mathrm{ml} / \mathrm{min} / \mathrm{Kg})$ at $1 \mathrm{M}, 30 \pm 2$ at $3 \mathrm{M}$ and $32 \pm 3$ at $6 \mathrm{M}$. Both parameters in the chronic phase increased significantly compared with those at $1 \mathrm{M}(\mathrm{p}<0.002$ and $p<0.0001)$. Thus, change in exercise capacity would correlate with change in left ventricular function.

Key words: acute myocardial infarction, cardiopulmonary exercise testing, myocardial scintigraphy
\end{abstract}

(J Jpn Phys Ther Assoc 6: 19-24, 2003)

E exercise testing (CPX) can evaluate non-invasively the respiratory-circulatory response. Furthermore, damaged sites and prognosis can be speculated from changes of each index ${ }^{1-3)}$. These indices are defined by many factors including left ventricular function, vasomotor tone, and muscle pump function by skeletal muscle, and oxygen application efficiency in the peripheral tissue ${ }^{4)}$. It has been reported that these factors have a complex relationship with each other and the mode of commitment can be affected by

Received: January 18, 2002

Accepted: September 9, 2002

Correspondence to: Hiroshi Maruoka, Department of Physical Therapy, School of Health and Social Services, Saitama Prefectural University, Saitama 343-8540, Japan the causes and the level of left ventricular pump dysfunction ${ }^{4) 5}$.

However, extension of the infarcted area and expansion of the non-infarcted area, which was named as remodeling (RM), in patients with acute myocardial infarction (AMI) affected patients' prognosis ${ }^{6)}$. Factors affecting RM are infarct size, infarct location, transmurality of the infarction, the extent of myocardial stunning, and the patency of the infarction-related artery ${ }^{6}$. However, there are few reports analyzing the relationship between change of left ventricular function and change of exercise capacity ${ }^{7-9)}$.

Thus, we measured left ventricular function in patients with AMI in their acute and chronic phases with a newlydeveloped and highly-reproducible single-photon- emission computed tomography (SPECT). We analyzed the 
Table 1. Clinical backgrounds

\begin{tabular}{lcc}
\hline Acute myocardial infarction & Male 34, Female 1 \\
Age (years) & $55.5 \pm 7.1$ \\
Infarction (n) & Anterior 25 \\
& Inferior 4 \\
& Lateral 6 \\
CAG/PTCA (n) & \multicolumn{2}{c}{$35 / 34$} \\
Vessel disease number (n) & (Pre PTCA) & (Post PTCA) \\
& OVD: 0 & OVD: 17 \\
& 1VD: 28 & 1VD: 14 \\
& 2VD: 5 & 2VD: 3 \\
Max. CPK (MB) & 3VD: 2 & 3VD: 0 \\
\hline
\end{tabular}

CAG: coronary angiography, PTCA: percutaneous transluminal coronary angioplasty, OVD: 0 vessel disease, 1VD: 1 vessel disease, 2VD: 2 vessel disease, 3VD: 3 vessel disease, Max. CPK: maximum creatine phosphokinase

relationship among left ventricular pump function, remodeling, and exercise capacity.

\section{Methods}

\section{Objective}

The subjects consisted of thirty five patients with first AMI (34 males and 1 female; mean age $55.5 \pm 7.1$ years) who were hospitalized in the Saitama Cardiovascular and Respiratory Center (Table 1). The infarct-related arteries were confirmed by urgent coronary angiography (CAG) on admission. Thirty-four of 35 patients were treated by percutaneous transluminal coronary angioplasty (PTCA) immediately after CAG. The remaining patient was not treated by PTCA because of a peripheral lesion of the coronary artery. The infarction sites were as follows: 25 for anterior, 6 for lateral and 4 for inferior.

\section{Protocol}

The study procedure is shown in Fig. 1. Briefly, PTCA was performed on admission (Day 1) and acute-phase dual, betamethyl-p-iodophenyl-pentadecanoic acid (BMIPP) and thallium (Tl), myocardial scintigraphy was performed on Day $4.9 \pm 2.2$. Chronic-phase myocardial scintigraphy with 99m Tc-tetrofosmin was performed on Day $188.5 \pm 22.9$. CPX was performed one month (1M, Day 31.4 \pm 9.0$)$, three months (3M, Day $95.9 \pm 22.8)$, and six months (6M, Day $184.0 \pm 43.7)$ after onset.

\section{Myocardial scintigraphy}

For dual myocardial scintigraphy, $74 \mathrm{MBq}$ of BMIPP and $111 \mathrm{MBq}$ of $\mathrm{Tl}$ were administered intravenously at rest and fasting in the morning. Scintigraphy was performed by electrocardiogram (ECG) gated SPECT 15 minutes after

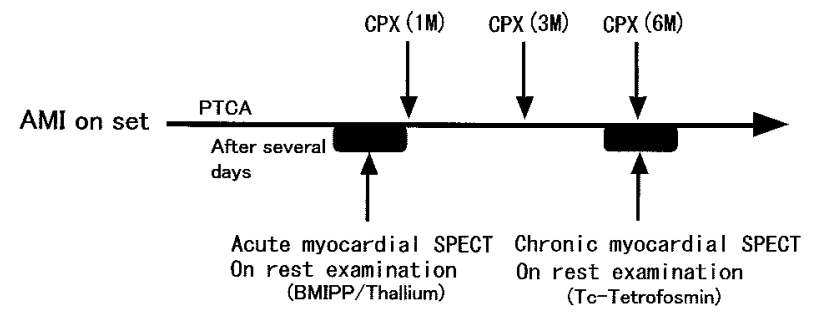

Fig. 1. Study protocol. AMI: acute myocardial infarction, PTCA: Percutaneous transluminal coronary angioplasty, SPECT: single photon emission computed tomography, CPX: Cardiopulmonary exercise testing.

injection. The SPECT system has a triple-headed gamma camera (PRISM3000) equipped with a low-energy collimator. Each detector rotated up to $120^{\circ}$ and imaging was performed every $6^{\circ}$. Data were acquired for 25 seconds per projection, and R-R interval on ECG was divided into 16 frames for the ECG gated method.

\section{Evaluation of left ventricular function}

Tl ECG gated SPECT was processed by a multipurpose computer and the left ventricular tomogram was reconstructed. Based on a gated short axis image, end diastolic volume (EDV), end systolic volume (ESV) and ejection fraction (EF) were obtained as parameters of left ventricular function by processing with Quantitative Gated SPECT (QGS) analyzing software. EDV, ESV and EF were also obtained in the chronic phase by quantitative gated SPECT with ${ }^{99 \mathrm{~m}}$ Tc-tetrofosmin.

\section{Left ventricular remodeling}

Left ventricular remodeling was defined as the presence of more than $10 \%$ dilatation of either end-systolic or end-diastolic left ventricle volume in the chronic phase compared with those in the acute phase. Subjects were divided into two groups by the presence or absence of left ventricular remodeling: RM group $(n=14)$ and non-RM group $(\mathrm{n}=21)$ (Table 2).

\section{Cardiopulmonary exercise testing (CPX)}

CPX was measured by the ramp loading method as a symptom limit. A treadmill was used as a tool for exercise loading, and a protocol in which loading linearly increases every minute after a 3-minute warm-up period was used. A stress system (Marquette) was used during exercise loading, with heart rate, ST-T changes, and arrhythmia monitored by a 12-lead ECG, and blood pressure measured every minute by STBP-780 (Nihon Colin). Criteria for the termination of a loading test included leg fatigue, dyspnea, severe arrhythmia, more than $2 \mathrm{~mm} \mathrm{ST}$-segments depression, blood pressure rise, chest pain, and heart rate predicted by age. 
Table 2. Clinical backgrounds (Remodeling group or Nonremodeling group)

\begin{tabular}{|c|c|c|c|}
\hline & & RM group & non-RM group \\
\hline \multicolumn{2}{|c|}{ Acute myocardial infarction (n) } & 14 & 21 \\
\hline \multirow[t]{3}{*}{ Infarction (n) } & Anterior & 11 & 14 \\
\hline & Inferior & 1 & 3 \\
\hline & Lateral & 2 & 4 \\
\hline \multicolumn{2}{|l|}{ CAG/PTCA (n) } & $14 / 14$ & $21 / 20$ \\
\hline \multirow{4}{*}{\multicolumn{2}{|c|}{ Vessel disease number (n) }} & 0VD:7 & 0VD: 10 \\
\hline & & 1VD: 7 & 1VD: 8 \\
\hline & & 2VD: 0 & 2VD: 3 \\
\hline & & 3VD: 0 & 3VD: 0 \\
\hline \multirow[t]{2}{*}{ Max. CPK (MB) } & IU/L & $6117 \pm 3035$ & $3710 \pm 2045$ \\
\hline & & $(564 \pm 286)$ & $(283 \pm 160)$ \\
\hline
\end{tabular}

RM: remodeling, non-RM: non-remodeling, Abbreviations as in Table 1.

Expiration gas analysis was performed by a breath-bybreath method using the heart lung function measuring system (CPX/D, Med Graphics Co.). The anaerobic threshold (AT) was determined mainly by the V-slope method in reference to the changes in ventilation equivalent against either oxygen uptake or carbon dioxide discharge quantity. Peak oxygen uptake $\left(\right.$ Peak $\left.\mathrm{V}_{2}\right)$ was defined as the average value during the last 30 seconds of loading and was used, together with AT, as indices of exercise capacity.

\section{Statistics}

Diachronic changes of AT, Peak $\dot{\mathrm{VO}}_{2}, \mathrm{EDV}, \mathrm{ESV}$, and EF were subjected to one-way layout variance analysis and tested using Bonferron's method. The relationship between AT and Peak $\dot{\mathrm{VO}}_{2}, \mathrm{EDV}, \mathrm{ESV}$, or EF was tested using Spearman's correlation analysis. All results are expressed as the mean \pm standard deviation (SD) and judged significant with a risk rate less than $5 \%$.

\section{Results}

Serial changes in heart rate and systolic blood pressure during cardiopulmonary exercise testing

The end point of the CPX was either a blood pressure rise or leg fatigue. The heart rate at the rest between the RM group and the non-RM group showed no significant difference. The heart rate at the end point between the RM group and the non-RM group also showed no significant difference. The systolic blood pressure between the RM group and the non-RM group showed no difference except for that at the rest 6 months after onset $(p<0.05)$ (Table 3$)$.

\section{Left ventricular volume and exercise capacity}

In the RM group, EDV and ESV were $105 \pm 24(\mathrm{ml})$ and $65 \pm 23$ in the acute phase and $147 \pm 4$ and $88 \pm 45$ in the chronic phase. Both parameters increased from acute phase to chronic phase. EF was $39 \pm 10(\%)$ in the acute phase and $42 \pm 12$ in the chronic phase. EF did not change significantly from acute phase to chronic phase. In the nonRM group, EDV and ESV were $106 \pm 28$ and $58 \pm 22$ in the acute phase and $113 \pm 28$ and $51 \pm 20$ in the chronic phase. Neither parameter changed significantly from acute phase to chronic phase. EF in the chronic phase $(55 \pm 7)$ was higher than in the acute phase $(46 \pm 10)$ (Table 4$)$.

In the RM group, AT and Peak $\mathrm{VO}_{2}$ were $14 \pm 2(\mathrm{ml} /$ $\mathrm{min} / \mathrm{Kg})$ and $24 \pm 4(\mathrm{ml} / \mathrm{min} / \mathrm{Kg})$ at $1 \mathrm{M}, 15 \pm 3$ and $27 \pm 5$ at $3 \mathrm{M}$, and $16 \pm 2$ and $26 \pm 4$ at $6 \mathrm{M}$, indicating no significant changes in these parameters between those at $1 \mathrm{M}$ and those at $3 \mathrm{M}$ and $6 \mathrm{M}$. In the non-RM group, AT was $15 \pm 1$ at $1 \mathrm{M}, 16 \pm 2$ at $3 \mathrm{M}$, and $18 \pm 4$ at $6 \mathrm{M}$, suggesting a significant increase at $3 \mathrm{M}$ and $6 \mathrm{M}(\mathrm{p}<0.002)$ compared with that at $1 \mathrm{M}$. Peak $\dot{\mathrm{VO}}_{2}$ was $26 \pm 3$ at $1 \mathrm{M}, 30 \pm 2$ at $3 \mathrm{M}$, and $32 \pm 3$ at $6 \mathrm{M}$, suggesting a significant increase at $3 \mathrm{M}$ and $6 \mathrm{M}(\mathrm{p}<0.0001)$ compared with that at $1 \mathrm{M}$. These results thus indicated that the change in exercise capacity correlated with left ventricular function (Table 4).

Table 3. Serial changes in heart rate and systolic blood pressure during cardiopulmonary exercise testing

\begin{tabular}{|c|c|c|c|c|c|c|c|}
\hline & & \multicolumn{3}{|c|}{ RM group } & \multicolumn{3}{|c|}{ non-RM group } \\
\hline & & 1 month & 3 months & 6 months & 1 month & 3 months & 6 months \\
\hline \multirow{2}{*}{$\begin{array}{l}\text { Heart rate } \\
\text { (beat } / \mathrm{min} \text { ) }\end{array}$} & Rest & $66.7 \pm 8.1$ & $68.1 \pm 6.6$ & $64.9 \pm 8.1$ & $69.9 \pm 9.8$ & $68.7 \pm 8.3$ & $68.0 \pm 6.4$ \\
\hline & Peak & $139.5 \pm 13.0$ & $147.8 \pm 17.3$ & $150.6 \pm 18.1$ & $145.5 \pm 12.2$ & $156.8 \pm 15.4$ & $158.0 \pm 14.3$ \\
\hline $\begin{array}{l}\text { Systolic } \\
\text { blood } \\
\text { pressure }\end{array}$ & Rest & $121.5 \pm 17.8$ & $130.9 \pm 14.1$ & $135.3 \pm 15.3$ & $124.6 \pm 11.5$ & $127.2 \pm 15.2$ & $124.5 \pm 10.7$ \\
\hline$(\mathrm{mmHg})$ & Peak & $167.6 \pm 37.2$ & $185.6 \pm 26.1$ & $194.1 \pm 28.3$ & $171.1 \pm 26.5$ & $186.6 \pm 27.9$ & $188.4 \pm 22.2$ \\
\hline
\end{tabular}

RM: remodeling. Cardiopulmonary test was performed 1 month, 3 and 6 months after the onset of acute myocardial infarction. $* \mathrm{p}<0.05$. 
Table 4. Chnage of left ventricular function and exercise capacity

\begin{tabular}{|c|c|c|c|c|c|}
\hline \multirow[t]{7}{*}{ RM group } & & \multicolumn{2}{|l|}{ Acute phase } & \multicolumn{2}{|c|}{ Chronic phase } \\
\hline & $\mathrm{EVD}(\mathrm{ml})$ & $105 \pm 24$ & \multicolumn{2}{|c|}{$147 \pm 41$} & \\
\hline & $\mathrm{ESV}(\mathrm{ml})$ & $65 \pm 23$ & \multicolumn{2}{|c|}{$88 \pm 45$} & \\
\hline & $\mathrm{EF}(\%)$ & $39 \pm 10$ & \multicolumn{2}{|c|}{$42 \pm 12$} & \\
\hline & & 1 month & 3 months & 6 months & \\
\hline & $\mathrm{AT}(\mathrm{ml} / \mathrm{min} / \mathrm{kg})$ & $14 \pm 2$ & $15 \pm 3$ & $16 \pm 2$ & $\mathrm{~ns}$ \\
\hline & Peak $\dot{\mathrm{V}} \mathrm{O}_{2}(\mathrm{ml} / \mathrm{min} / \mathrm{kg})$ & $24 \pm 4$ & $27 \pm 5$ & $26 \pm 4$ & ns \\
\hline \multirow[t]{7}{*}{ Non-RM group } & & \multicolumn{2}{|l|}{ Acute phase } & \multicolumn{2}{|c|}{ Chronic phase } \\
\hline & $\operatorname{EVD}(\mathrm{ml})$ & \multicolumn{2}{|l|}{$106 \pm 28$} & $113 \pm 28$ & \\
\hline & $\mathrm{ESV}(\mathrm{ml})$ & \multicolumn{2}{|l|}{$58 \pm 22$} & $51 \pm 20$ & \\
\hline & $\mathrm{EF}(\%)$ & $46 \pm 10$ & \multicolumn{2}{|c|}{$55 \pm 7$} & \\
\hline & & 1 month & 3 months & 6 months & \\
\hline & $\mathrm{AT}(\mathrm{ml} / \mathrm{min} / \mathrm{kg})$ & $15 \pm 1$ & $16 \pm 2$ & $18 \pm 4$ & $\mathrm{p}<0.002$ \\
\hline & Peak $\dot{\mathrm{VO}}_{2}(\mathrm{ml} / \mathrm{min} / \mathrm{kg})$ & $26 \pm 3$ & $30 \pm 2$ & $32 \pm 3$ & $\mathrm{p}<0.0001$ \\
\hline
\end{tabular}

RM: remodeling, EDV: end diastolic volume, ESV: end systolic volume, EF: ejection fraction, AT: anaerobic threshold, Peak $\dot{\mathrm{VO}}_{2}$ : peak oxygen uptake.

Gated SPECT (EDV and ESV Images)

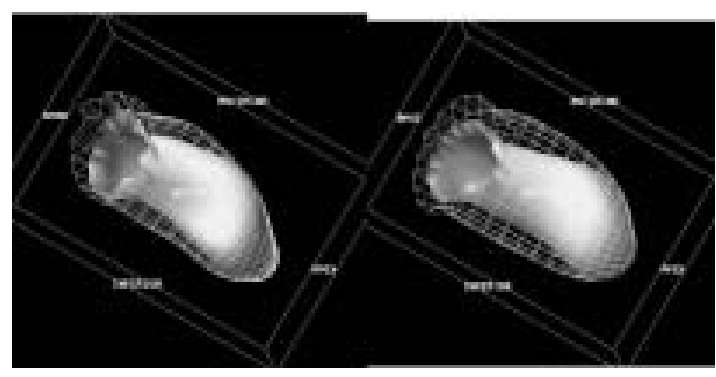

Acute phase

Chronic phase
Gated SPECT (Volumes)

\begin{tabular}{l|cc} 
& $\begin{array}{c}\text { Acute } \\
\text { phase }\end{array}$ & $\begin{array}{c}\text { Chronic } \\
\text { phase }\end{array}$ \\
\hline $\operatorname{EDV}(m \mathrm{l})$ & 115 & 148 \\
$\operatorname{ESV}(\mathrm{ml})$ & 60 & 70 \\
$\operatorname{EF}(\%)$ & 48 & 53
\end{tabular}

53

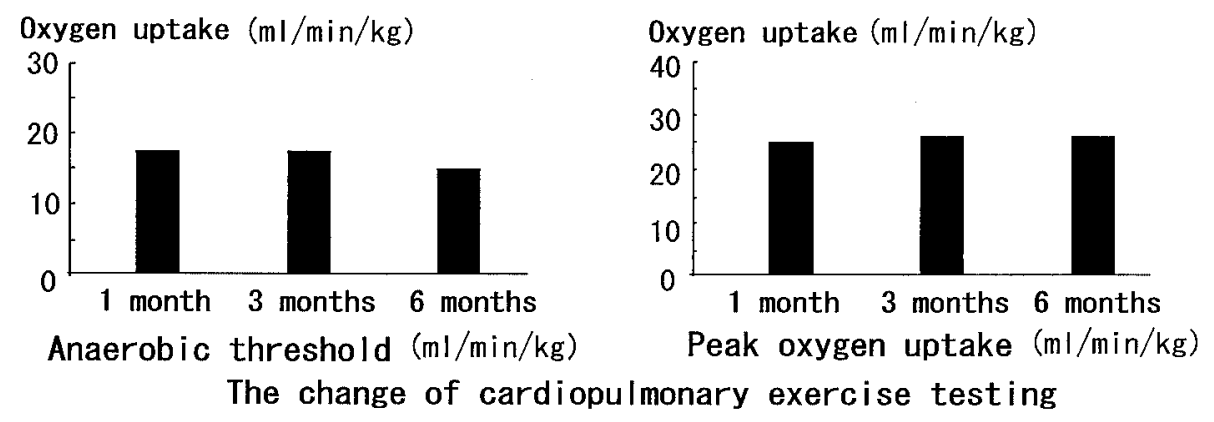

Fig. 2. Case presentation: remodeling case. EDV: end diastolic volume, ESV: end systolic volume, EF: ejection fraction, SPECT: single photon emission computed tomography.

\section{Relationship between left ventricular function and exercise} capacity

EDV, ESV, and EF in the chronic phase showed significant correlation with Peak $\dot{\mathrm{VO}}_{2}$ at $6 \mathrm{M}(\mathrm{r}=-0.35$, $<0.05 ; \mathrm{r}=-0.49, \mathrm{p}<0.004 ; \mathrm{r}=0.50, \mathrm{p}<0.004$, respectively). Except for these, there was no significant correlation among the other parameters.

\section{Case presentations}

Patient with remodeling (52-year-old male) (Fig. 2): CAG shows total occlusion in the central side of the left anterior descending artery. EDV and ESV increased from the acute phase to the chronic phase. AT and Peak $\dot{\mathrm{V}}_{2}$ at $1 \mathrm{M}, 3 \mathrm{M}$ and $6 \mathrm{M}$ did not change significantly.

Patient without remodeling (45-year-old male) (Fig. 3): 


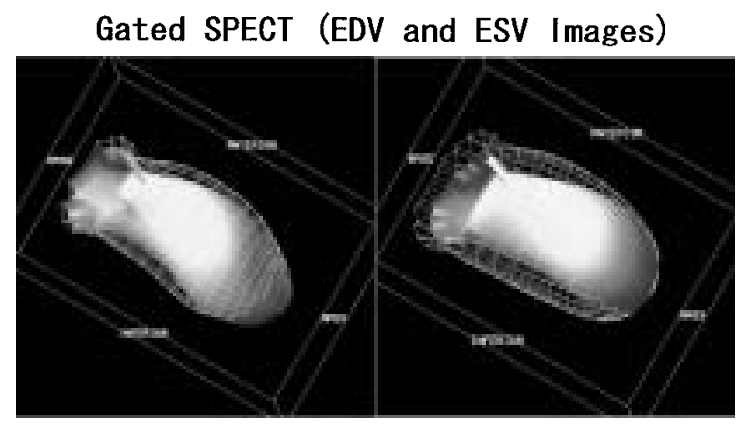

\begin{tabular}{|c|c|c|}
\hline \multicolumn{3}{|c|}{ Gated SPECT (Volumes) } \\
\hline & $\begin{array}{l}\text { Acute } \\
\text { phase }\end{array}$ & $\begin{array}{c}\text { Chronic } \\
\text { phase }\end{array}$ \\
\hline $\operatorname{EDV}(\mathrm{ml})$ & 120 & 120 \\
\hline $\operatorname{ESV}(\mathrm{m} I)$ & 77 & 64 \\
\hline $\mathrm{EF}(\%)$ & 36 & 47 \\
\hline
\end{tabular}

Acute phase

Chronic phase

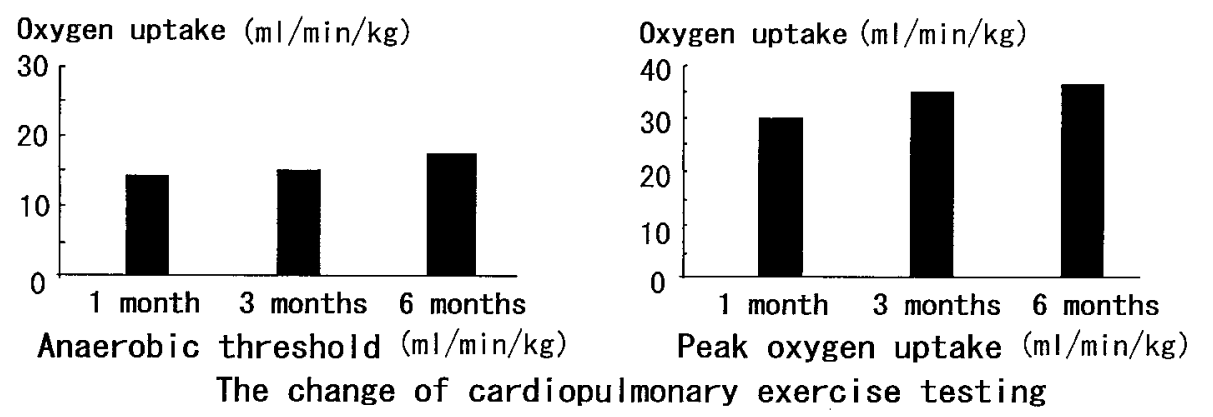

Fig. 3. Case presentation: non-remodeling case. EDV: end diastolic volume, ESV: end systolic volume, EF: ejection fraction, SPECT: single photon emission computed tomography.

CAG shows total occlusion in the central side of the left anterior descending artery and was diagnosed AMI. ESV decreased from the acute phase to the chronic phase. The AT and Peak $\dot{\mathrm{VO}}_{2}$ at $3 \mathrm{M}$ and $6 \mathrm{M}$ were greater than those at $1 \mathrm{M}$.

\section{Discussion}

The purpose of this study was to analyze the relationship among left ventricular pump function, remodeling, and exercise capacity. The analysis of the relationship would predict left ventricular function recovery. Furthermore, this relationship would be clinically important for exercise prescription, such as the setting exercise strength, and prediction of patients' prognoses. The results from the current study show that there were no significant changes in the RM group at $\mathrm{AT}$ and Peak $\mathrm{VO}_{2}$ at $1 \mathrm{M}$ compared, with those at $3 \mathrm{M}$ and $6 \mathrm{M}$. In contrast, they increased significantly at $3 \mathrm{M}$ and $6 \mathrm{M}$ compared with those at $1 \mathrm{M}$ in the non- $\mathrm{RM}$ group.

\section{Relationship between remodeling and exercise capacity}

Exercise capacity in patients with AMI is regulated by both central factors and peripheral factors ${ }^{10)}$. Thus, improvement of left ventricular function is important for increase in exercise capacity. Left ventricular function itself is one of the regulatory factors for exercise capacity. Furthermore, the mechanism of the relationship between exercise capacity and left ventricular function has been reported as follows ${ }^{10) 11)}$. An increase in cardiac output is chiefly attributable to the Frank-Starling effect due to an increase in preload at exercise with loading less than AT. However, at exercise with loading more than AT, it is brought by increased contraction strength.

However, myocardium has a response with a number of compensation mechanisms against continuous loading. In patients with AMI in particular, the loss in contractile components of myocardium would be attributable to the loading to the remnant myocardium. It has been reported that increasing the contractility of non-infarcted myocardium and increased diastolic volume contributes to the Frank-Starling effect would preserve systemic blood perfusion ${ }^{12) 13)}$. Such morphological and functional adaptation of myocardium in patients with AMI is a compensatory reconstruction and these changes are named as remodeling ${ }^{6}$. It has also been reported that patients with progressive cardiomegaly had a higher mortality rate ${ }^{14)}$.

In this study, we analyzed the relationship between remodeling and exercise capacity. No significant changes were observed in AT and Peak $\mathrm{VO}_{2}$ between those at $1 \mathrm{M}$ and those at $3 \mathrm{M}$ and $6 \mathrm{M}$ in the RM group, whereas they increased significantly from $1 \mathrm{M}$ to $3 \mathrm{M}$ or $6 \mathrm{M}$ in the nonRM group. These results indicate that left ventricular function would be correlative by evaluating changes in exercise capacity.

Taniguchi et al. reported that the indices of exercise capacity at $1 \mathrm{M}$ did not correlate with rest left ventricular function, whereas diachronic increment of exercise capacity might correlate weakly with left ventricular function ${ }^{15)}$. 
Murabayashi and his group reported that the exercise capacity in patients with AMI is regulated by both central and peripheral factors; improvement of the left ventricular function and improvement of the muscle volume ${ }^{3)}$. Therefore, the change in exercise capacity was conceived a prescription factor of the cardiac output during exercise. The current results show the relationship between changes of exercise capacity and left ventricular function.

\section{Relationship between left ventricular function and exercise capacity}

It has been reported that exercise capacity correlates with either causes or levels of left ventricular pump dysfunction ${ }^{4}$. In particular, systolic and diastolic function of the left ventricular and right ventricular function would be important parameters. In the patient with AMI, accumulation of lactic acid in active muscle due to decreased blood flow would be attributable to the general reduction of left ventricular contractile function. This decreased contractile function would induce a loss of exercise capacity ${ }^{16) 17)}$.

Relationship between left ventricular function and exercise capacity was investigated in this study. The results presented show the significant relationship between EDV, ESV, and EF in the chronic phase with Peak $\dot{\mathrm{VO}}_{2}$ at $6 \mathrm{M}$, but no relationship was observed among other factors, such as that between EDV, ESV, and EF in the acute phase with Peak $\dot{\mathrm{VO}}_{2}$ at $1 \mathrm{M}$ or $3 \mathrm{M}$. Measuring left ventricular function was performed at rest generally. Murayama commented that left ventricular function at rest is not reflected in exercise capacity ${ }^{18)}$. Baker and his colleagues ${ }^{8)}$ and Taniguchi et al. ${ }^{15)}$ concluded that exercise capacity does not reflect to rest left ventricular function because there are no relationship between exercise capacity and EF and cardiac index that are acquired by left ventriculography. The condition measuring left ventricular function in this study was at rest. Exercise capacity is an index of exertion. The different condition of measurement might be one of the responsible factors for the absence of relationship between left ventricular function and exercise capacity. Further studies would be required to understand these points.

\section{References}

1) Florea VG, Henein MY, Anker SD, et al:: Prognostic value of changes over time in exercise capacity and echocardiographic measurements in patients with chronic heart failure. Eur Heart J 21: 146-153, 2000.

2) Karel P, Johan VC, Johan V, et al.: Peak oxygen uptake better predicts outcome than submaximal respiratory data in heart transplant candidates. Circulation 101: 1152-1157, 2000.

3) Murabayashi $T$, Itoh $\mathrm{H}$, Kato $\mathrm{M}$, et al: : Mechanisum of increase exercise tolerance in patients with acute myocardial infarction. J Cardiol 30: 241-249, 1997 (in Japanese).

4) Katou M, Itou H: Factor of decides in the exercise tolerance. Cardiologist 2: 309-313, 1997 (in Japanese).

5) Asano R, Sumiyoshi T: Left ventricular dysfunction as a predictor of survival after recovery from myocardial infarction. Kokyu to jyunkan 43: 661-669, 1995 (in Japanese).

6) Sutton J MG, Sharpe N: Left ventricular remodeling after myocardial infarction pathophysiology and therapy. Circulation 101: 2981-2988, 2000.

7) Nakayama K: Sequential changes in the left ventricular function during ramp exercise in patients with ischemic heart disease. Kakuigaku 30: 1081-1090, 1993 (in Japanese).

8) Baker BJ, Wilen MM, Boyd LM, et al:: Relation of right ventricular ejection fraction to exercise capacity in chronic left ventricular failure. Ame J Cardiol 54: 596-599, 1984.

9) Kitzman DW, Higginbotham MB, Cobb FR, et al.: Exercise intolerance in patients with heart failure and preserved left ventricular systolic function-failure of the Frank Starling mechanism. JACC 17: 1065-1072, 1991.

10) Yoshikawa J (ed): Practical Cardiology 3, Bunkodou, Tokyo, 1996 pp 198-208 (in Japanese).

11) Koike A, Hiroe M, Adachi $\mathrm{H}$, et al.: The relation of the oxygen uptake and cardiac output in the exercise load in heart disease patients. Coronary 25: 81-82, 1993 (in Japanese).

12) Takao $\mathrm{Y}$, Hayasaki $\mathrm{K}$, Shono $\mathrm{H}$, et al.: Compensatory mechanisms and the remodeling of the left ventricle after acute myocardial infarction evaluated by digital subtraction angiography. Coronary 24: 13-21, 1992 (in Japanese).

13) Ibukiyama $T$ : The left ventricle after acute myocardial infarction. Coronary 24: 22-23, 1992 (in Japanese).

14) Giannuzzi P, Tavazzi L, Temporelli PL, et al.: Long term physical training and left ventricular remodeling after anterior myocardial infarction. JACC 22: 1821-1829, 1993.

15) Taniguchi K: Cardiopulmonary Exercise Testing. Nankodo, Tokyo, 1993, pp 338-347 (in Japanese).

16) Lejemtel $\mathrm{TH}$, Maskin CS, Lucido $\mathrm{D}$, et al: Failure to augment maximal limb blood flow in response to one leg versus two leg exercise in patients with severe heart failure. Circulation 74: 245, 1986.

17) Conley AJ, Stainer K, Rowley JM, et al.: Abnormalities of the peripheral circulation and respiratory function in patients with severe heart failure. Brt Heart J 55: 75, 1986.

18) Murayama M (ed): Cardiology NOW10-Exercise Guidance Exercise Treatment. Nankodo, Tokyo, 1995, pp 50-51 (in Japanese). 